\title{
CONCORDANCIA EN EL ESTUDIO DE RETINOGRAFÍAS EN DIABÉTICOS: OFTALMÓLOGOS VS MÉDICOS DE FAMILIA
}

\section{AGREEMENT AMONG OPHTHALMOLOGISTS AND PRIMARY CARE PHYSICIANS IN THE EVALUATION OF RETINOGRAPHIES OF DIABETIC PATIENTS}

\author{
ANDONEGUI J ${ }^{1}$, BERÁSTEGUI L ${ }^{1}$, SERRANO L ${ }^{2}$, EGUZKIZA A ${ }^{3}$, GAMINDE I ${ }^{4}$, ALISEDA D ${ }^{5}$
}

\section{RESUMEN}

Objetivo: Determinar la concordancia en la interpretación de retinografías no midriáticas de pacientes diabéticos entre oftalmólogos y un grupo de médicos de atención primaria previamente sometidos a un proceso de adiestramiento.

Métodos: El estudio fue dividido en dos fases. En la primera se adiestró a los participantes en la interpretación de imágenes retinográficas. La segunda fase se realizó sobre 1000 imágenes correspondientes a 200 pacientes, 100 sin retinopatía diabética y 100 con retinopatía. Los participantes debían decidir si las imágenes presentaban o no signos de retinopatía diabética. Para la valoración de la concordancia se utilizó el índice kappa. Se estimó como adecuado un porcentaje de desacuerdo del $15 \%$ con una precisión del $5 \%( \pm 5 \%)$ con un nivel de confianza del $95 \%$.

Resultados: El porcentaje de diagnósticos coincidentes entre oftalmólogos y médicos de atención primaria está entre 89 y 97,5\%. Por lo que respecta

\begin{abstract}
Purpose: To assess the extent of agreement in the evaluation of non-mydriatic retinographies of diabetic patients among ophthalmologists and a group of primary care physicians with previous training.

Methods: The study was divided in two phases. In the first phase, the four participants were instructed in the interpretation of retinographies. The second phase involved the evaluation of 1000 images of 200 patients, 100 without retinopathy and 100 with signs of diabetic retinopathy. The four participants had to decide if the images did or did not show evidence of diabetic retinopathy. Kappa index was used to assess the extent of agreement. A percentage disagreement of $15 \%$ with a precision of $5 \%$ $( \pm 5 \%)$ with a confidence level of $95 \%$ was considered adequate.

Results: The percentage of coincident diagnoses among ophthalmologists and primary care physicians was between 89 and $97.5 \%$. With respect to the assessment of the agreement, the kappa index
\end{abstract}

\footnotetext{
Recibido: 31/5/07. Aceptado: 14/8/08.

1 Licenciado en Medicina.

5 Doctor en Medicina. del Gobierno de Navarra. España.

Correspondencia:

José Andonegui

Servicio de Oftalmología. Hospital de Navarra

31008 Pamplona (España)

España

E-mail: jandonen@cfnavarra.es
}

Servicio de Oftalmología. Hospital de Navarra. Pamplona. España.

2 Profesor Titular. Departamento de Ingeniería Eléctrica y Electrónica. Universidad Pública de Navarra. Navarra. España.

3 Ingeniero Técnico de Telecomunicaciones. Departamento de Ingeniería Eléctrica y Electrónica. Universidad Pública de Navarra. Navarra. España.

${ }^{4}$ Licenciada en Sociología. Dpto. de Docencia e Investigación. Gobierno de Navarra. España.

Financiado parcialmente mediante un proyecto de investigación (modalidad Beca Mikel Larumbe Zazu) concedido por el Departamento de Salud 
a la evaluación de la concordancia, el índice kappa se sitúa entre 80 y $95 \%$. En todos los casos el intervalo de confianza incluye el $85 \%$.

Conclusiones: Después de un adecuado adiestramiento, la fiabilidad de facultativos de atención primaria en interpretar retinografías no midriáticas de diabéticos es muy alta. Esto permitiría establecer en atención primaria el filtro para el cribado de la retinopatía diabética. Las ventajas de esta medida serían una mayor implicación de atención primaria en el manejo global de los diabéticos y una disminución de la demanda de atención oftalmológica.

Palabras clave: Retinopatía diabética, retinografía no midriática, cribado, atención primaria.

\section{INTRODUCCIÓN}

La prevalencia de la diabetes mellitus en los países desarrollados se sitúa en torno al 6-8\% de la población. Una de las complicaciones más temidas de esta enfermedad es la retinopatía diabética, cuyas consecuencias para la visión pueden ser devastadoras. Para prevenir y tratar de forma temprana las alteraciones provocadas en la retina por la diabetes, la mayor parte de las autoridades recomiendan una revisión anual del fondo de ojo (1). Pero la elevada prevalencia de esta enfermedad en los países de nuestro entorno hace que, en la práctica, un elevado porcentaje de estos pacientes no puedan acudir a ser revisados dentro de los plazos indicados. Para intentar solucionar este problema, algunos autores proponen la utilización de los sistemas de retinografía no midriática para la exploración del fondo de ojo (2). Hasta ahora, se ha considerado que la interpretación de las imágenes obtenidas mediante los sistemas de retinografía no midriática debía ser llevada a cabo por oftalmólogos. El objetivo de este trabajo es valorar la concordancia en la interpretación de retinografías no midriáticas de pacientes diabéticos entre oftalmólogos y un grupo de médicos de atención primaria previamente sometidos a un proceso de adiestramiento.

\section{SUJETOS, MATERIAL Y MÉTODOS}

Para llevar a cabo este estudio se seleccionó a cuatro médicos de atención primaria que habían demostrado una gran implicación en acciones formativas y was between 80 and $95 \%$. In all cases the confidence interval was at least $85 \%$.

Conclusions: After an adequate training process, the reliability of evaluation of non-mydriatic retinographies of diabetic patients by primary care physicians was very high. This could allow the establishment of screening for diabetic retinopathy at the primary care level. Advantages of this system include a greater involvement of primary care physicians in the global management of diabetic patients and a lower demand for ophthalmic attention (Arch Soc Esp Oftalmol 2008; 83: 527-532).

Key words: Diabetic retinopathy, non-mydriatic retinography, screening, primary care.

una elevada predisposición a procesos de innovación en las tareas asistenciales. El proceso fue dividido en dos fases, una primera de adiestramiento teórico y una segunda de evaluación de la concordancia. A su vez, la fase de adiestramiento se subdividió en otras dos fases, una primera de tipo presencial y una segunda «on-line». La parte de formación presencial consistió en dos sesiones teóricas de dos horas de duración cada una en las cuales a los participantes se les explicó detalladamente como era el aspecto de un fondo de ojo normal, cuales eran las principales alteraciones patológicas no relacionadas con la diabetes que podíamos encontrar en la retina y por último cuales eran los signos de cada uno de los estadíos de la retinopatía diabética.

La fase de formación «on-line» fue realizada de forma individual por cada uno de los participantes. Al acabar el adiestramiento presencial se les entregó un CD que contenía, en primer lugar, archivos escritos e imágenes demostrativas de los temas tratados. Además, el CD incluía una parte de autoevaluación con 1000 imágenes correspondientes al fondo de ojo de 200 pacientes diabéticos, cinco imágenes de cada uno de los pacientes en diferentes campos. De los 200 pacientes, 100 no presentaban retinopatía diabética y los otros 100 tenían algún grado de retinopatía diabética. En cada uno de los pacientes, los médicos de atención primaria debían optar entre dos opciones, no retinopatía diabética o retinopatía diabética. No se fijó como objetivo el determinar el grado de retinopatía. Los participantes tenían la opción de consultar en cualquier momento el diagnóstico de cada uno de los casos. Los resultados de los diagnósticos emitidos se iban acu- 
mulando en una base de datos incluida en el CD de forma que al acabar toda la serie se podía comprobar el porcentaje de aciertos. Este proceso de autoevaluación podía repetirse todas las veces que se considerase necesario.

Cuando los participantes estimaban que su grado de adiestramiento era adecuado, se pasaba a la última fase, la evaluación de la concordancia. Para la misma, el CD incluía otras 1000 imágenes de 200 pacientes diabéticos. De ellas, también 100 eran de pacientes sin retinopatía y 100 de pacientes con algún grado de retinopatía diabética. El orden de las imágenes era diferente para cada uno de los cuatro participantes. Igual que en la autoevaluación, en cada caso se debía determinar si había o no retinopatía diabética. En esta fase, a diferencia de lo que ocurría en la autoevaluación, el CD no permitía consultar los diagnósticos. Las respuestas emitidas se almacenaban en otra base de datos que los participantes debían enviar, una vez finalizada la serie, a los coordinadores de este estudio para valorar la concordancia. Se estimó como adecuado un porcentaje de desacuerdo del $15 \%$ con una precisión del $5 \%( \pm 5 \%)$ con un nivel de confianza del $95 \%$. Esta estimación de un índice de concordancia (Kappa) del $85 \%$ es considerada casi perfecta en la clasificación de Landis y Koch. Para evitar algunos de los problemas atribuidos al índice kappa (que está afectado por la prevalencia del problema), se utilizaron un $50 \%$ de retinografías normales y un $50 \%$ de retinografías patológicas.

Las imágenes fueron obtenidas con un Retinografo no Midriático TOPCON modelo TRC NW6S.

\section{RESULTADOS}

El número de respuestas coincidentes con lo diagnosticado por oftalmólogos entre los 200 casos oscila entre $195(97,5 \%)$ y $178(89 \%)$. El número de falsos positivos está entre $1(0,5 \%)$ y $14(7 \%)$. El de falsos negativos oscila entre $4(2 \%)$ y $13(6,5 \%)$.
Uno de los participantes dejo sin evaluar dos de los casos presentados (1\%), los otros tres evaluaron los 200 casos. Los resultados aparecen expuestos en la tabla I.

Por lo que respecta a la evaluación de la concordancia, el índice kappa se sitúa entre 80 y 95\%. En todos los casos el intervalo de confianza incluye el $85 \%$. Los resultados aparecen expuestos en la tabla II.

\section{DISCUSIÓN}

La elevada prevalencia de diabetes en los países de nuestro entorno hace que la revisión anual del fondo de ojo por parte de un oftalmólogo a todos los pacientes diabéticos exceda las posibilidades de asistencia de la mayor parte de los sistemas sanitarios. Para intentar paliar este problema en los últimos años se han introducido como alternativa frente a las consultas de oftalmología los sistemas de retinografía no midriática (2). Estos sistemas presentan varias ventajas, entre ellas, que resultan más económicos, emplean menos tiempo por paciente, pueden ser aplicados a poblaciones físicamente alejadas de los centros de atención especializada y no precisan dilatación pupilar. La fiabilidad de estos sistemas para detectar las alteraciones provocadas por la retinopatía diabética es muy similar a la de los métodos clásicos de exploración retiniana (3-5). También se ha valorado la concordancia existente entre la interpretación de las imágenes digitales de la retina y la exploración directa de la misma en las consultas de oftalmología y se ha encontrado que en general la correlación entre ambos sistemas es muy

Tabla II. Evaluación de la concordancia

Índice Kappa

Intervalo de confianza

\begin{tabular}{lll}
\hline Participante 1 & $95 \%$ & $90-99 \%$ \\
Participante 2 & $93 \%$ & $80-98 \%$ \\
Participante 3 & $81 \%$ & $73-89 \%$ \\
Participante 4 & $80 \%$ & $72-88 \%$ \\
\hline
\end{tabular}

Tabla I. Resultados de la interpretación de las retinografías

\begin{tabular}{lccccccc}
\hline Participante & $\begin{array}{c}\text { Diagnósticos } \\
\text { correctos }\end{array}$ & $\begin{array}{c}\% \text { diagnósticos } \\
\text { correctos }\end{array}$ & $\begin{array}{c}\text { Falsos } \\
\text { positivos }\end{array}$ & $\begin{array}{c}\% \text { falsos } \\
\text { positivos }\end{array}$ & $\begin{array}{c}\text { Falsos } \\
\text { negativos }\end{array}$ & $\begin{array}{c}\% \text { Falsos } \\
\text { negativos }\end{array}$ & $\begin{array}{c}\text { No } \\
\text { valorados }\end{array}$ \\
no valorados
\end{tabular}


alta, lo que avalaría la utilidad del sistema de retinografía no midriática como método de cribado y detección de la retinopatía diabética (6-9). Por otra parte, existen estudios que han demostrado que la retinografía no midriática es superior en la relación coste-efectividad para la detección de la retinopatía diabética si se compara con los métodos tradicionales de exploración retiniana (10).

Se estima que la prevalencia de Retinopatía Diabética en la población puede estar alrededor de un 25\% (11). En un análisis preliminar que se efectuó de los primeros 400 pacientes revisados por oftalmólogos de nuestro servicio mediante el sistema de retinografía no midriática, se encontró que la prevalencia de retinopatía diabética era del $12 \%$ (datos no publicados). Esta baja prevalencia podría ser debida a que muchos de los pacientes con afectación retiniana o bien ya están siendo controlados en las consultas de oftalmología o bien aquejan alteraciones visuales, en cuyo caso no se incluyen para ser valorados mediante retinografía no midriática. Este alto porcentaje de pacientes con fondos de ojo clasificados como normales nos hizo plantearnos el que fueran los facultativos de atención primaria quienes, tras un proceso de adiestramiento, se encargasen de evaluar las retinografías.

Generalmente se considera que debe ser un oftalmólogo la persona encargada de interpretar las imágenes retinianas obtenidas con los sistemas de retinografía no midriática. Aunque existen algunos estudios en los que se ha evaluado la concordancia en la interpretación de imágenes retinianas en la retinopatía diabética entre oftalmólogos y médicos de atención primaria, en ninguno de ellos se ha utilizado como método de exploración la retinografía no midriática $(12,13)$. El porcentaje de falsos negativos encontrados en el primero de estos estudios fue de un $27,3 \%$ (12), mientras que en el segundo los autores describen un porcentaje de falsos negativos de un $13 \%$ (13). El porcentaje de falsos negativos obtenido por nosotros oscila entre el 2 y el $6,5 \%$. Los falsos negativos son especialmente relevantes puesto que se trata de pacientes cuyos fondos son descritos como normales pero que presentan algún grado de retinopatía diabética. En el segundo de los trabajos, que también estudia la concordancia mediante el índice kappa, los autores obtienen unos valores que oscilan entre 6 y 70 (13), inferiores y con mucha mayor variabilidad que los de nuestro estudio. Pensamos que la mayor concordancia y homogeneidad lograda por nuestros participantes pueden ser debidas a que el método de aprendizaje empleado, que a diferencia de los otros incluye una prolongada formación «on-line», puede considerarse como más exhaustivo.

El objetivo de este trabajo era demostrar que los médicos de atención primaria, después de un entrenamiento adecuado, pueden diferenciar con una fiabilidad muy alta un fondo de ojo normal de uno con algún signo de retinopatía diabética. Los resultados obtenidos avalan nuestro planteamiento, puesto que el nivel de concordancia obtenido por los cuatro participantes, una vez efectuado el correspondiente análisis mediante el índice kappa, se sitúa dentro de los niveles que nos habíamos fijado como adecuados. Se puede considerar, por tanto, que con un entrenamiento similar al empleado en nuestro estudio, médicos de atención primaria pueden distinguir con una elevada fiabilidad las diferencias entre un fondo de ojo normal y un fondo de ojo con retinopatía diabética en las imágenes obtenidas mediante un sistema de retinografía no midriática.

La aplicación clínica de estos datos sería establecer en atención primaria el filtro para el cribado de la retinopatía diabética. Tras haber formado mediante el procedimiento descrito a una serie de médicos de familia, aquellos pacientes sin signos de retinopatía diabética en las retinografías podrían continuar siendo revisados anualmente por los facultativos encargados de este sistema, mientras que las retinografías que mostrasen algún grado de retinopatía serían remitidas para ser evaluadas por oftalmólogos. Un porcentaje muy elevado de los pacientes que son revisados anualmente mediante retinografía no midriática presentan fondos de ojo que son etiquetados como normales. Conseguir que los oftalmólogos no tuviesen que verse obligados a revisar un gran número de fondos de ojo sin alteraciones patológicas permitiría que pudiesen dedicar más tiempo a pacientes con problemas de mayor relevancia, lográndose de esta manera un importante aumento de la eficiencia de las consultas y una clara optimización de los recursos. Además, establecer en atención primaria el cribado de la retinopatía diabética permite aumentar la implicación de los médicos de familia en el manejo integral de los pacientes diabéticos y consolidar la exploración del fondo de ojo como una más de las actuaciones preventivas en estos enfermos. De esta manera, un importante número de pacientes podrán ser controlados durante periodos más o menos largos de tiempo en su centro de salud. 
En conclusión, los médicos de atención primaria después de un adecuado proceso de adiestramiento pueden diferenciar con una fiabilidad muy alta un fondo de ojo normal de uno con signos de retinopatía diabética en las imágenes obtenidas mediante sistemas de retinografía no midriática. Esto puede permitir establecer en el nivel de atención primaria el filtro para el cribado de la retinopatía diabética. Las ventajas de esta medida serían una mayor implicación de los médicos de familia en el manejo total de sus pacientes diabéticos y una disminución de la demanda en las consultas de oftalmología.

\section{BIBLIOGRAFÍA}

1. American Diabetes Association. Standards of medical care for patients with diabetes mellitus. Diabetes Care 2000; 23: S32-S42.

2. Hernáez-Ortega MC, Soto Pedre E, Vázquez, JA, Gutiérrez. MA, Asua J. Estudio de la eficiencia de una cámara no midriática en el diagnóstico de Retinopatía Diabética. Rev Clin Esp 1998; 198: 194-199.

3. Stellingwerf C, Hardus PL, Hooymans JM. Two-field photography can identify patients with vision-threatening diabetic retinopathy: a screening approach in the primary care setting. Diabetes Care 2001; 24: 2086-2090.

4. Baeza Díaz M, Gil Guillén V, Orozco Beltrán D, Pedrera Carbonell V, Ribera Montes C, Pérez. Pons I, et al. Validez de la cámara no midriática en el cribado de la retinopatía diabética y análisis de indicadores de riesgo de la retinopatía. Arch Soc Esp Oftalmol 2004; 79: 433-442.

5. Lin DY, Blumenkranz MS, Brothers RJ, Grosvenor DM. The sensitivity and specificity of single-field nonmydriatic monochromatic digital fundus photography with remote image interpretation for diabetic retinopathy screening: a comparison with ophtalmoscopy and standardized mydriatic color photography. Am J Ophthalmol 2002; 134: 204-213.

6. Ahmed J, Ward TP, Bursell SE, Aiello LM, Cavallerano $J D$, Vigersky MD. The sensitivity and specificity of nonmydriatic digital stereoscopic retinal imaging in detecting diabetic retinopathy. Diabetes Care 2006; 29: 2205-2209.

7. Gómez-Ulla F, Fernández MI, González F, Rey P, Rodríguez, M, Rodríguez-Cid MJ, et al. Digital retinal images and teleophthalmology for detecting and grading diabetic retinopathy. Diabetes Care 2002; 25: 1384-1389.

8. Massin P, Aubert JP, Eschwege E, Erginay A, Bourovitch $J C$, BenMehidi A, et al. Evaluation of a screening program for diabetic retinopathy in a primary care setting Dodia (Déspistage ophtalmologique du diabète) study. Diabetes Metab 2005; 31: 153-162.

9. López-Bastida J, Cabrera-López F, Serrano-Aguilar P. Sensitivity and specificity of digital retinal imaging for screening diabetic retinopathy. Diabet Med 2007; 24: 403-407.

10. Sender Palacios MJ, Montserrat Bagur S, Badia Llanch X, Maseras Bover M, De la Puente Martorell ML, Foz Sala M. Cámara de retina no midriática: estudio de coste-efectividad en la detección temprana de la retinopatía diabética. Med Clin (Barc) 2003; 121: 446-452.

11. Goldaracena MB, Escudero JM, Arrondo A, Villarrubia A, Armendía B, Iturralde R. Prevalencia de retinopatía diabética en una población diabética registrada en atención primaria. Arch Soc Esp Oftalmol 1998; 73:263-268.

12. Baeza Díaz M, Pedrera Carbonell V, Reigadas López R, Orozco Beltrán D, Soler Ferrández F, Miralles Gisbert S. Concordancia en la exploración de fondo de ojo en el cribaje de la retinopatía diabética entre el médico de familia y el oftalmólogo. Arch Soc Esp Oftalmol 1999; 74: 465-472.

13. Gill JM, Cole DM, Lewobitz HM, Diamond JJ. Accuracy of screening for diabetic retinopathy by family physicians. Ann Fam Med 2004; 2: 218-220. 